\title{
Abnormal Wave Propagation in Passive Media
}

\author{
M. Mojahedi, Member, IEEE, K. J. Malloy, Member, IEEE, G. V. Eleftheriades, Senior Member, IEEE, \\ J. Woodley, Member, IEEE, and R. Y. Chiao
}

\begin{abstract}
Abnormal velocities in passive structures such as one-dimensional (1-D) photonic crystals and a slab having a negative index of refraction are discussed. In the case of 1-D photonic crystal, the frequency- and time-domain experiments for waves tuned to the bandgap of the photonic crystal demonstrate a positive group velocity exceeding the speed of light in vacuum (superluminal). In the case of a medium with negative index of refraction, our theoretical studies show that such a medium can support positive group and negative phase velocities (backward waves), as well as negative group and negative phase velocities. The meaning of superluminal group velocity and negative group velocity, or equally, positive superluminal group delay and negative group delay, are discussed. It is shown that despite their counterintuitive meaning there are no contradictions with the requirements of relativistic causality (Einstein causality). To clearly demonstrate this, the important subject of the "front" is reintroduced.
\end{abstract}

Index Terms-Meta materials, negative group velocity, negative index of refraction, superluminal group velocity.

\section{INTRODUCTION}

$\mathbf{T}$ HE FACT that the group velocity of an electromagnetic wave packet (pulse) can exceed the speed of light in vacuum (described as "superluminal") has been demonstrated in experiments at microwave frequencies [1]-[7], at optical frequencies [8], and in the single-photon limit [9], [10]. As a starting point, an interested reader may consult the review by Chiao and Steinberg [11]. Despite one's initial impression, superluminal group velocities are not at odds with the requirements of relativistic causality (Einstein causality), and indeed, it can be shown that they must exist as a consequence of the Kramers-Kronig relations, which in themselves are a statement of the system linearity and causality [12]-[15].

The point that in the regions of anomalous dispersion group velocity can become abnormal was first considered by Sommerfeld and his student Brillouin [16]. In their studies, they examined a sinusoidally modulated step function propagating through a medium with Lorentz-Lorenz dispersion. They iden-

\footnotetext{
Manuscript received September 18, 2002; revised December 10, 2002. This work was supported by the National Aeronautics and Space Administration under Grant NRA-99-LeRC-1.

M. Mojahedi, G. V. Eleftheriades, and J. Woodley are with the Electrical and Computer Engineering Department, University of Toronto, Toronto, ON, M5S 3G4 Canada (e-mail: mojahedi@waves.utoronto.ca).

K. J. Malloy is with Center for High Technology Materials, Department of Electrical Engineering and Computer Engineering, University of New Mexico, Albuquerque NM 87106 USA.

R. Y. Chiao is with the Department of Physics, University of California at Berkeley, Berkeley, CA 94720 USA.

Digital Object Identifier 10.1109/JSTQE.2002.807971
}

tified and defined phase, group, energy, and "front" ${ }^{1}$ velocities. However, for reasons unknown to the authors, while the first three velocities have received much attention in both undergraduate and graduate textbooks, the "front" velocity and the closely related concept of the first or Sommerfeld forerunner has not enjoyed the same status. This is even more surprising since, among the aforementioned velocities, it is only the "front" velocity alone that must satisfy the requirements of Einstein causality. In other words, Einstein causality does not always equate the group velocity with the velocity of information transfer, particularly when propagation of "attenuated traveling waves" 2 is involved.

Interestingly, while abnormal velocities have received much attention within the "physics community," and even have been discussed in the new edition of the well-respected text book by Jackson [18, pp. 325-326], the "engineering community" is less aware of these developments. Ironically, this occurs in spite of the fact that some of the earliest work in this subject was first published in the microwave journals [19]-[21]. Unfortunately, the cited work suffered from many misunderstandings and misinterpretations and was the subject of controversy for sometime. For example, the authors in [19]-[21] did not describe their work in terms of evanescent wave propagation, which is the underlying physical mechanism for the observed superluminal group velocities in passive medium and confused the notions of phase, group, "front", and information velocities, implying that they have transmitted "radio messages faster than light." Interestingly, under appropriate circumstances, the experimental setup used by the aforementioned authors has been used by others to demonstrate the superluminal group velocities in undersized waveguides or slightly misaligned horn antennas [4], [6], [22], [23].

In this paper, the term "abnormal velocity" is used to identify two general categories. In one case, the group velocity is positive and exceeds the speed of light in vacuum (superluminal), and in the other case, the group velocity and, hence the group delay, is negative. As an example of the former case, we experimentally study the wave propagation through the bandgap of a one-dimensional photonic crystal (1-DPC), in both the frequency and time domain. In the frequency domain we rely on measuring the transmission phase and the group delay, and it is observed that the group delay for the attenuated traveling waves propagating through the 1-DPC, while positive, is less than the length of the

\footnotetext{
${ }^{1}$ To be complete, one has to add the term "signal velocity" defined as the velocity of the half-maximum point to the above list. However, by their own admission, such a definition is arbitrary and as discussed in [1] can become superluminal.

${ }^{2}$ We have used the term "attenuated traveling waves" in the same sense as in [17], although sometimes the term evanescent is used to signify the same thing.
} 
1-DPC divided by $c$, hence implying a superluminal group velocity. In the time-domain experiment, the time-of-flight for a single tunneling wave packet tuned to the bandgap of the 1-DPC is directly compared to a wave packet traveling an equal distance in air (vacuum).

Wave propagation through a medium with negative index of refraction (NIR) is an example of the second category of the abnormal velocities, in which the group velocity and group delay can be negative. In light of the recent interests in such metamaterials, also sometimes referred to as the left-handed-media (LHM), and the confusion surrounding the meaning, necessity, and existence of the negative group velocity for these metamaterials [24]-[28], a systematic study of this subject is warranted. In Section IV, we theoretically study the wave propagation through a slab or multiple slabs having an NIR. It is observed that such media can in principle support both negative phase and group velocities in addition to positive group and negative phase velocities, i.e., backward waves [29, pp. 263].

In light of the experimental and theoretical evidences for superluminal and negative group velocities presented here and elsewhere, a question remains to be answered. How do these anomalous behaviors comply with the principles of special relativity which require that no signal (information) travel faster than $c$. To answer this question the important subject of "front" is reintroduced. It is argued that from a purely theoretical point of view, genuine information carried by an electromagnetic pulse (or pulses) is to be associated with the pulse "front" and the field oscillations immediately following the "front", i.e., the Sommerfeld forerunner.

This paper is organized as follows. In Sections II and III the experimental evidence of superluminal behavior in the frequency and time domain are presented. The subject of negative group velocity and wave propagation in a medium having an effective NIR is discussed in Section IV. The important topic of the "front" and its relation to the Einstein causality is discussed in Section V. Our final thoughts and remarks are summarized in Section VI.

\section{Measuring Superluminal Group Velocity in FREQUENCY DOMAIN}

Setup used to measure the group delay and hence the group velocity for a 1-DPC in the frequency domain is shown in Fig. 1.

In order to properly measure the superluminal group velocities in the frequency domain accurate and reliable knowledge of the transmission phase is necessary. For a noncoaxial or freespace setup shown in Fig. 1, the implementation of a conventional short-open-load and thru (SOLT) calibration is difficult, if not impossible [30]. Furthermore, in our measurement, it is pivotal to ensure that insertion losses and phase delays associated with the setup (particularly the antennas, waveguide to antennas transitions, and the air segments surrounding the 1-DPC) are removed, and a reference plane is established at a point between the two antennas and at their respective far-fields. To this end, a thru-reflect-line (TRL) calibration is used [31, pp. 217-222], [30], [32]. The "thru" standard is a free-space transmission line of the length $58.9 \mathrm{~cm}$, while the "line" standard is a free-space transmission line of the length $59.24 \mathrm{~cm}$, and the

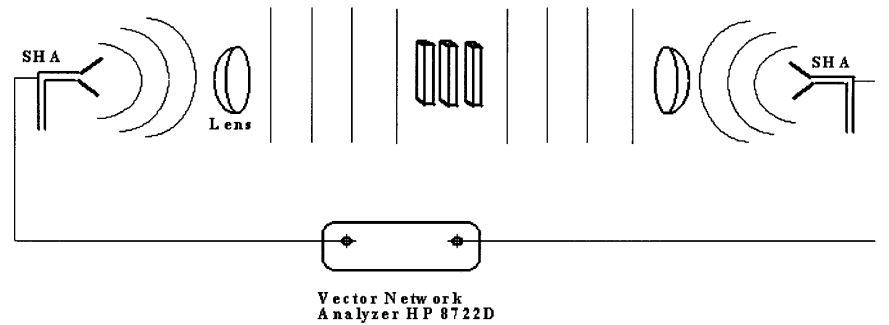

Fig. 1. Setup used in the frequency domain. It consists of a standard horn antenna (SHA), vector network analyzer (VNA), and collimating lenses.

"reflect" is a copper plate set midway between the transmitter and receiver. The accuracy and precision of the setup and the calibration scheme was tested by using the setup to measure the transmission function and consequently obtaining the index of refraction for slabs of Polycarbonate, Teflon, and PVC. At the respective frequency range (18 to $26 \mathrm{GHz}$ ), the comparison between the published results and our measurements were very good [33], implying the veracity of our technique.

By performing the TRL calibration, a reference plane of unit magnitude and zero phase for the transmission, at the midway point between the two standard horn antennas (SHA), was established. The 1-DPC was then inserted between the two collimating lenses, and the receiver antenna was moved backward a distance equal to the physical thickness of the 1-DPC $\left(L_{\mathrm{pc}}\right)$. Fig. 2(a) and (b) shows the measured and calculated transmission magnitudes and unwrapped phases for 1-DPC's with one, two, and three Eccostock ${ }^{\circledR}$ dielectric slabs $(N=1,2,3)$.

The thickness and the index of refraction for the dielectric slabs were $1.33 \mathrm{~cm}$ and $3.4-j 0.002$, respectively, whereas the air spacer region had a thickness of $1.76 \mathrm{~cm}$ and index of unity. The theoretical curves were obtained using the procedure described in [2].

We expect to observe the superluminal behavior for frequencies within the bandgap of the 1-DPC, for which in the limit of an infinitely long 1-DPC, the waves are evanescent and acquire no insertion phase as they propagate. Clearly, as the number of slabs is increased, our finite 1-DPC better approximates the infinitely long 1-DPC. At the same time, due to the strong attenuation of the transmitted signal [see Fig. 2(a)], it becomes more difficult to accurately and reliably measure the transmission magnitude and phase. Consequently, the comparison between the detected and calculated transmission functions worsens as the number of layers are increased.

The group delay and group velocity are related to the transmission phase $(\phi)$ according to

$$
\tau_{g}=-\frac{\partial \phi}{\partial \omega}=-\frac{\partial \operatorname{Arg}(T)}{\partial \omega}
$$

and

$$
\frac{v_{g}}{c}=\frac{L_{\mathrm{pc}}}{c \tau_{g}}
$$

where $T$ is the transmission function, $\omega$ is the radial frequency, and $L_{\mathrm{pc}}$ is given by

$$
L_{\mathrm{pc}}=(N-1)\left(d_{i}+d_{l}\right)+d_{i} .
$$

In (3), $d_{l}$ and $d_{i}$ are the dielectric thickness and spacer thickness, respectively. 


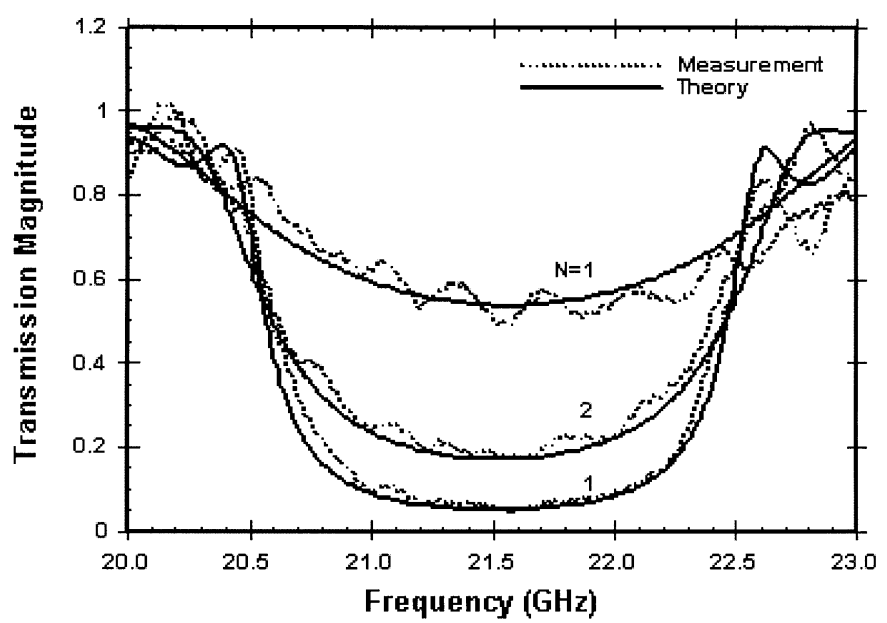

(a)

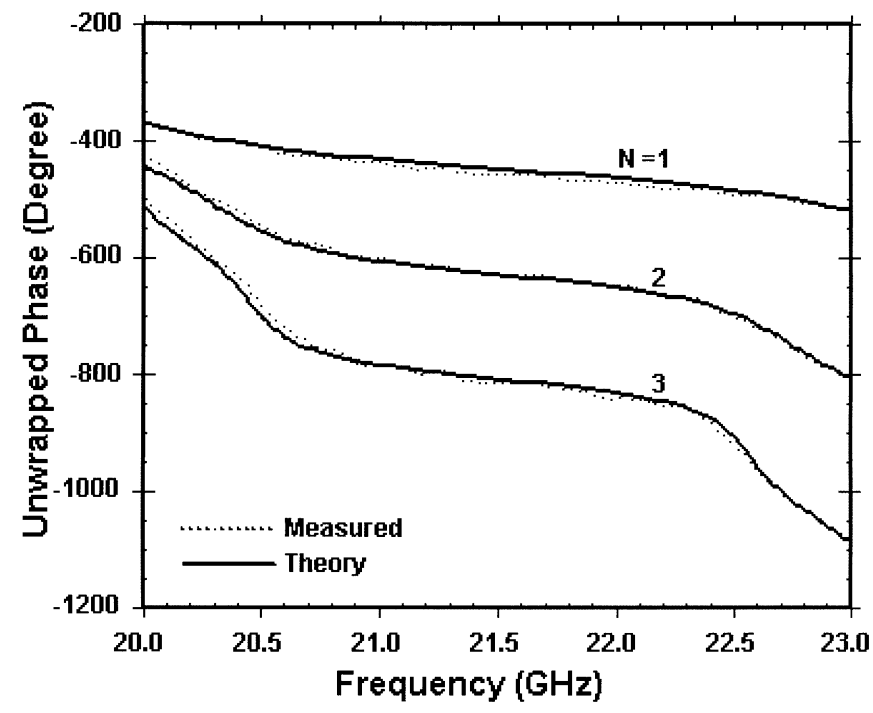

(b)

Fig. 2. Measured and calculated transmission magnitudes and unwrapped phases for the 1-DPC with one, two, or three, Eccostock dielectric slabs: (a) transmission magnitude and (b) transmission phase.

Equations (1) and (2) imply that in order to acquire the group delay and group velocity from the measured phase information, curves in Fig. 2(b) must be differentiated. However, differentiating noisy ${ }^{3}$ data is a challenging task, and smoothing the curves prior to differentiation is a notoriously arbitrary process that may introduce artificial results. To avoid this, we have obtained a nonlinear least square fit to the experimental phase data. The best least square fit to the data can be obtained as a function of the dielectric thickness $\left(d_{l}\right)$, spacer thickness $\left(d_{i}\right)$, and the real part of the Eccostock ${ }^{\circledR}$ index of refraction $\left(n_{l}\right)$. A Fortran program based on the IMSL subroutine DBCLSF, which uses a modified Levenberg-Marquardt algorithm and a finite-difference Jacobian, is used to obtain the best least square fit. Fig. 3 shows the result of the least square fit of the phase data together with applying (1), in order to determine the group delay $\left(\tau_{g}\right)$ for a 1-DPC with one, two, and three Eccostock dielectric slabs.

\footnotetext{
${ }^{3}$ Note that the vertical axis for the unwrapped phase in Fig. 2(b) spans a large range ( -200 to -1200 degree). Consequently, the effects of noise on the phase data is not immediately evident from Fig. 2(b). A closer examination of the wrapped phase for the frequencies within the stop-band, however, shows a small yet detectable variation of the phase.
}

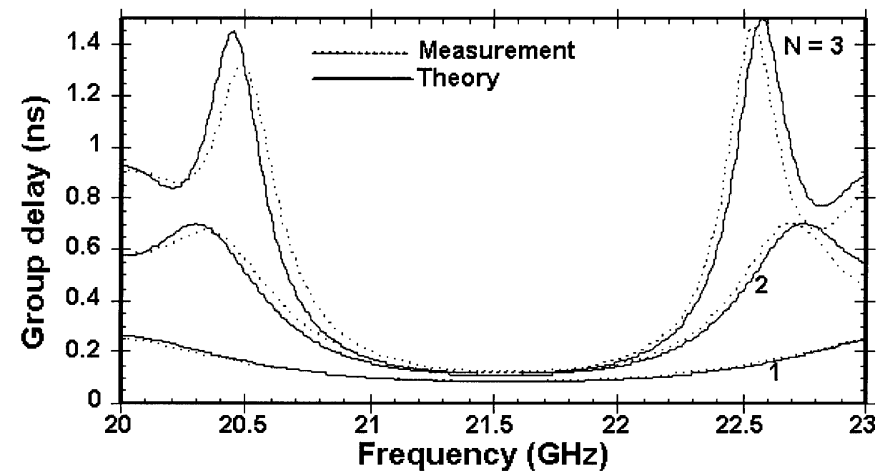

Fig. 3. Measured and calculated group delay for the 1-DPC. The parameters used to obtain the fitted curves (measurement) and the calculated curves (theory) are given in Table I.

TABLE I

FitTed AND MEASUREd PARAMETERS FOR 1-DPC With 1, 2 , AND 3 DIELECTRIC SLABS

\begin{tabular}{l|c|c|c|c}
\hline & $\begin{array}{c}\text { Fitting, } \\
N=3\end{array}$ & $\begin{array}{c}\text { Fitting, } \\
N=2\end{array}$ & $\begin{array}{c}\text { Fitting, } \\
N=1\end{array}$ & Measured \\
\hline$d_{i}$ & $1.794 \mathrm{~cm}$ & $1.825 \mathrm{~cm}$ & & $1.76 \mathrm{~cm}$ \\
\hline$d_{l}$ & $1.399 \mathrm{~cm}$ & $1.366 \mathrm{~cm}$ & $1.396 \mathrm{~cm}$ & $1.33 \mathrm{~cm}$ \\
\hline$n_{l}^{\prime}$ & 3.216 & 3.288 & 3.245 & 3.40 \\
\hline$n_{l}^{\prime \prime}$ & 0.002 & 0.002 & 0.002 & 0.002 \\
\hline
\end{tabular}

The fitting parameters for the case of $N=3,2,1$ and the measured values of these parameters are given in Table I.

Having acquired the group delay, the normalized group velocity can easily be obtained from (2) and (3). This is shown in Fig. 4, where in addition to velocities derived from the measurements (dotted curves), the theoretical values for group velocities are also shown (solid curves).

A closer examination of Fig. 4 reveals that while one dielectric slab $(N=1)$ is inadequate to produce superluminal group velocity, two slabs are sufficient to set up the interferences resulting in a group velocity exceeding $c$. In the case of $N=3$, a maximum superluminal group velocity 2.1 times $c$ is observed. Finally, for the normalized group velocities shown in Fig. 4 a detailed analysis of the experimental errors can be found in [33]. The conclusion of these analyses is the fact that the maximum error due to the experimental uncertainties associated with the index of refraction of the dielectric slab, the slab thickness, and the spacer thickness are less than 0.14 , with an error even smaller than 0.14 in the range for which maximum superluminal behavior is observed. In short, none of the above experimental uncertainties are large enough to place the depicted superluminal group velocities below the "light line."

From the theoretical and experimental results presented in this section, it is evident that for the frequencies within the bandgap of a passive structure such as 1-DPC, the group delay while positive (see Fig. 3) is less than the physical length of the 1-DPC divided by $c\left(0<\tau_{g}<L_{\mathrm{pc}} / c\right)$. This in turn implies a positive and superluminal group velocity for these frequencies $\left(V_{g}>c>0\right)$. In Section III, a setup is used to further measure the superluminal group velocity directly in the time domain. 


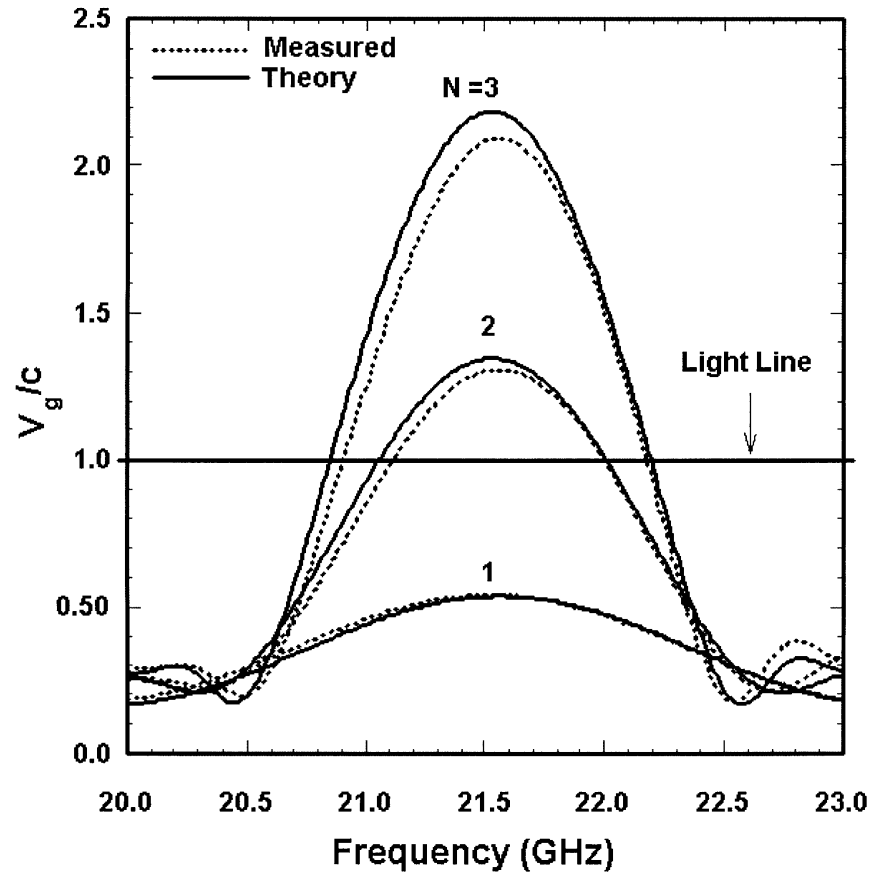

Fig. 4. Normalized group velocity for the 1-DPC. The dotted curves are the measured results obtained from the fitted curves in Fig. 3 and (2). The solid curves are theoretically calculated.

\section{Measuring Superluminal Group Velocity in Time DOMAIN}

In Section II, a frequency-domain setup was used to detect the superluminal group delays $\left(0<\tau_{g}<L_{\mathrm{pc}} / c\right)$ and group velocities for a 1-DPC. A more elegant approach is to observe this abnormal behavior directly in the time domain, verifying the fact that the envelope of a wave packet tuned to the stop-band of a photonic crystal propagates superluminally. To accomplish this, a 1-DPC consisting of five alternating layers of Polycarbonate $(n=1.66)$ and air $(n=1)$ was designed to have a stop-band at $9.68 \mathrm{GHz}$ (the center frequency of our source). Fig. 5 shows the experimental setup.

A backward wave oscillator (BWO) was used to generate the microwave pulse, and a mode converter (MC) was used to convert the $\mathrm{TM}_{01}$ mode of the BWO to a $\mathrm{TE}_{11}$. The pulse was then radiated via a conical horn antenna (CHA). The frequency output of the source was tuned to the mid-gap frequency of the 1-DPC at $9.68 \mathrm{GHz}$ (full-width at half-maximum (FWHM) of $100 \mathrm{MHz}$ ) and was detected by two HP 8470-B, Schottky diode detectors (provided in pair). Two single-channel Tektronix SCD-5000 fast oscilloscopes (4.5 GHz bandwidth) were used to display the pulse traces. In order to ensure that the two oscilloscopes were triggered as close to each other as possible, a line from the accelerator section of the BWO was connected to a fast Pico-second pulse generator, PSPL-model 4500E, which in turn was used to trigger the Tektronix scopes. The pulse generator was capable of producing trigger pulses with very sharp raise time (10\% to $90 \%$ rise time of roughly $100 \mathrm{ps}$ ) and consequently was used to ensure that in the worst case scenario the scopes were triggered within 20 ps from each other.

The CHA radiation intensity was sampled along two distinct directions (paths), referred to as "side" and "center." A series

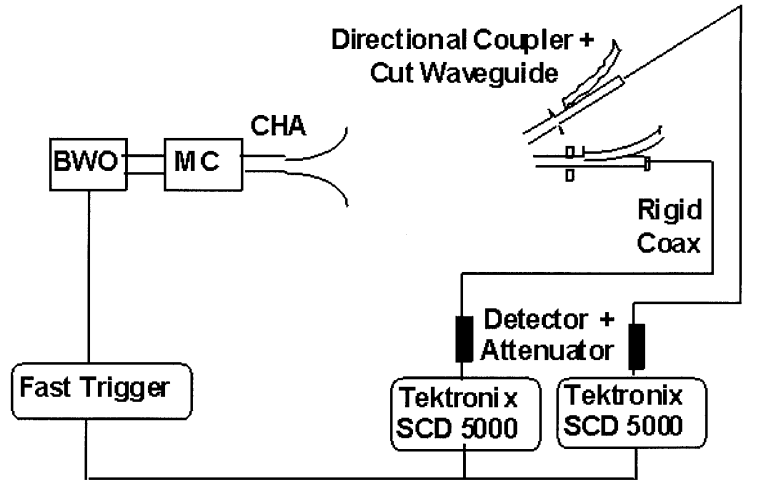

Fig. 5. Setup used in the time-domain experiment.

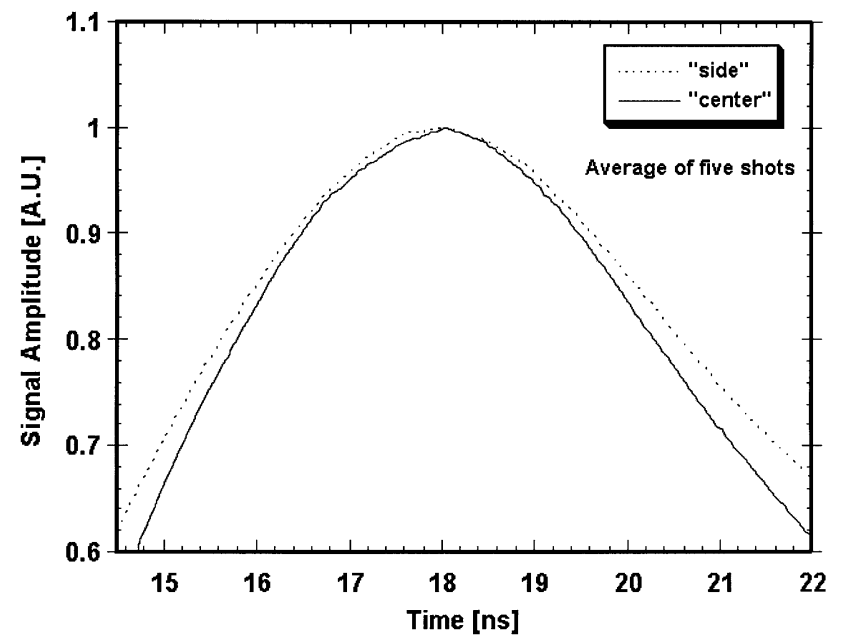

Fig. 6. The "center" and "side" pulses without the 1-DPC present. The pre-existing delays between the two paths are electronically removed such that the peaks of the two pulses arrive at the same time.

of microwave pulses were fired in order to measure and then remove the time-delay differences between the two paths due to differences in the cable lengths, internal detection of the oscilloscopes (Tektronix SCD 5000), and other incompatibilities. Fig. 6 shows the normalized ${ }^{4}$ wave packets after the setup delay has been compensated, such that the peaks corresponding to the pulses traveling through the "center" and "side" paths, in the absence of the 1-DPC, have arrived at the same time. At this point, the 1-DPC was inserted along the "center" path and a series of single pulses were fired. Fig. 7 shows the result for the average of five pulses. From the figure, it is clear that the peak of the wave packet propagating along the "center" path and tunneling through the 1-DPC has arrived $440 \pm 20 \mathrm{ps}$ earlier than the accompanying pulse propagating through the free-space along the "side" path. For a well-behaved wave packet, the theoretical value of the time-shift for the pulse peak can be obtained from (4) in which the $\tau_{g}$ is the group delay

$$
\Delta t=\frac{L_{\mathrm{pc}}}{c}-\tau_{g} .
$$

In the case of the 1-DPC studied here, the $L_{\mathrm{pc}}$ was $22.75 \mathrm{~cm}$ and the $\tau_{g}$ was calculated to be $320 \mathrm{ps}$ at $9.68 \mathrm{GHz}$. Using these values in (4), a theoretical value of $\Delta t=438 \mathrm{ps}$ was obtained which is in good agreement with the experimentally measured

\footnotetext{
${ }^{4}$ Each wave packet is normalized to its respective maximum amplitude.
} 


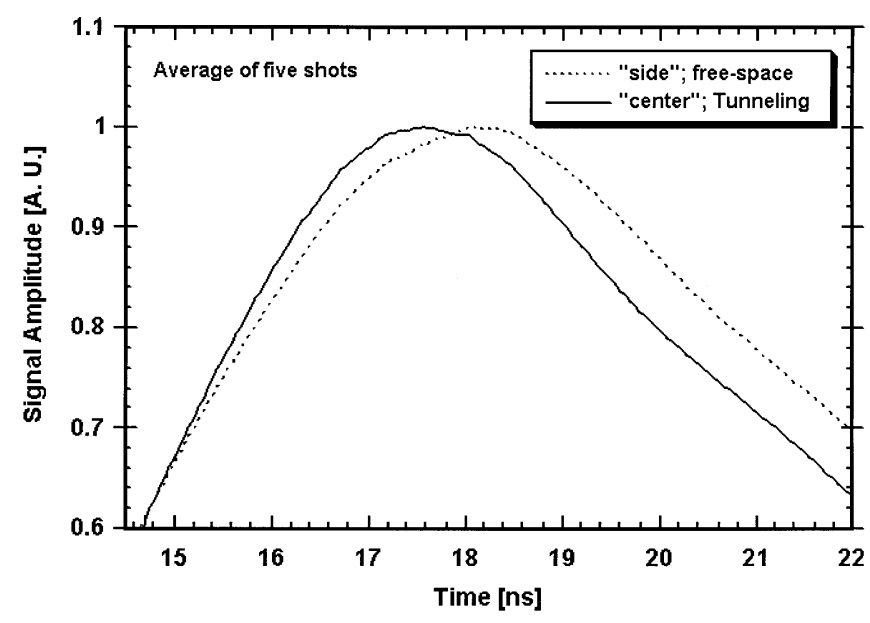

Fig. 7. The 1-DPC was inserted along the "center" path. The tunneling pulse ("center") has shifted to earlier times by $(440 \pm 20)$ ps.

value of $440 \pm 20$. The advancement in time for the tunneling pulse $(\Delta t)$ can easily be translated to a measure of the wave packet group velocity according to

$$
V_{g}=\frac{L_{\mathrm{pc}}}{\left(\frac{L_{\mathrm{pc}}}{c}\right)-\Delta t} .
$$

Equation (5) indicates that the tunneling wave packet propagated with a group velocity $(2.38 \pm 0.15) c$ as compared to the theoretically calculated value of $2.37 \mathrm{c}$.

In order to assure that the observed superluminal behavior is not the consequence of mere chance and random effects, the curves depicted in Figs. 6 and 7 are each the average of five pulses. However, in any averaging processes the point to be concerned about is the similarity (in terms of the mean) between the averaged quantity and its constituents. The quantity that best measures the similarities or differences between multiple sets of data is well known as the standard error [34, pp. 609-610], and for the data shown in Figs. 6 and 7 the maximum standard error was 0.0097 (corresponding to maximum variance of 0.09 ) for the curve labeled "side," depicted in Fig. 6. Clearly, the small magnitude of our maximum standard error implies that the constituents of our averaged curves closely resemble the resultant averaged curves.

The traditional point of view has commonly regarded the group velocity in the region of anomalous dispersion for which a large amount of attenuation and dispersion accompanies the wave packet, as an unphysical quantity. For example, Landau and Lifshitz state: "When considerable absorption occurs. The group velocity cannot be used, since in an absorbing medium wave packets are not propagated but rapidly ironed out" [35]. Brillouin expresses the same sentiment when stating “...but if absorption also occurs, a (the wave number) becomes complex or imaginary and the group velocity ceases to have a clear physical meaning" [36, pp. 75].

For this reason, it is important to compare the waveforms prior to and after tunneling in the experiment described above. Fig. 8 shows that in contrast to this common belief, the tunneling wave packet of Fig. 7 suffers minimal dispersion such that the FWHM of the pulse after tunneling was only increased by $1.5 \%$. In obtaining this figure, the tunneling wave packet was

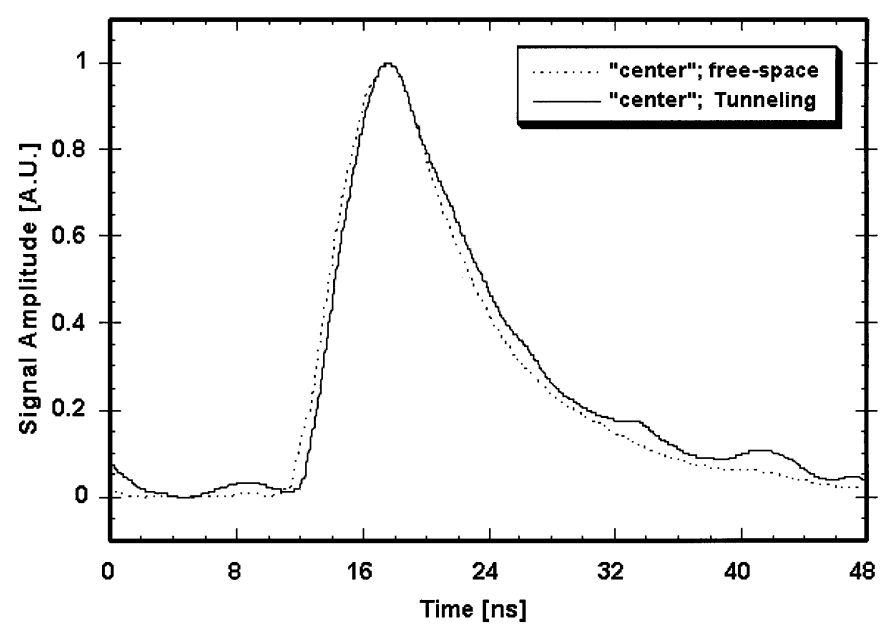

Fig. 8. A comparison between the tunneling and the free-space pulse propagating along the "center" path. The tunneling pulse has been manually shifted to later times in order to make the comparison easier.

manually moved to later times in order to make the comparison between the two pulses easier. The reader must note that, for the purpose of comparison, the curves in Figs. 6-8 are normalized to their respective maximum (note the arbitrary units). However, in terms of the actual units the maximum amplitude of the curve labeled "center", Tunneling in Figs. 7 and 8, has been reduced by a factor of 2.8 as compared to the maximum amplitude of the curve labeled "center" in Fig. 6. This reduction in amplitude is the result of evanescent propagation of the tunneling wave packet through the bandgap of the 1-DPC.

The physical meaning of superluminal group velocities discussed in Sections II and III can be understood as the following. For a well-behaved wave packet propagating through a medium capable of supporting superluminal group velocities, the peak of the wave packet (although reduced in magnitude) always arrives sooner than a similar wave packet traveling the same distance in vacuum. In the cases studied so far, the group velocity and group delay are positive and superluminal. In Section IV, we consider the second category of the abnormal velocities for which the group velocity and group delay are negative.

\section{Negative Group Velocity in Meta Materials}

For the second category of our abnormal velocities, we consider the case of negative group velocity and negative group delay (a group advance). With the recent possibilities in manufacturing a media having a negative index of refraction (NIR), also referred to as LHM or meta-materials [25]-[27], [37], [38], the subject of negative group velocity deserves some attention.

Unfortunately, this subject suffers from misunderstandings and misconceptions, particularly as to the connection between LHM and negative group velocities. For example, it has been suggested that a negative group velocity is a necessary signature of the LHM [25] and has been stated that the term LHM is to be understood as substances with negative group velocities [24] and that the left-handed medium has a band with negative group velocity [26]. On the other hand, experimental and theoretical studies presented thus far only consider the case of antiparallel phase and group velocities (backward waves) for which the group velocity is positive and points away from the 


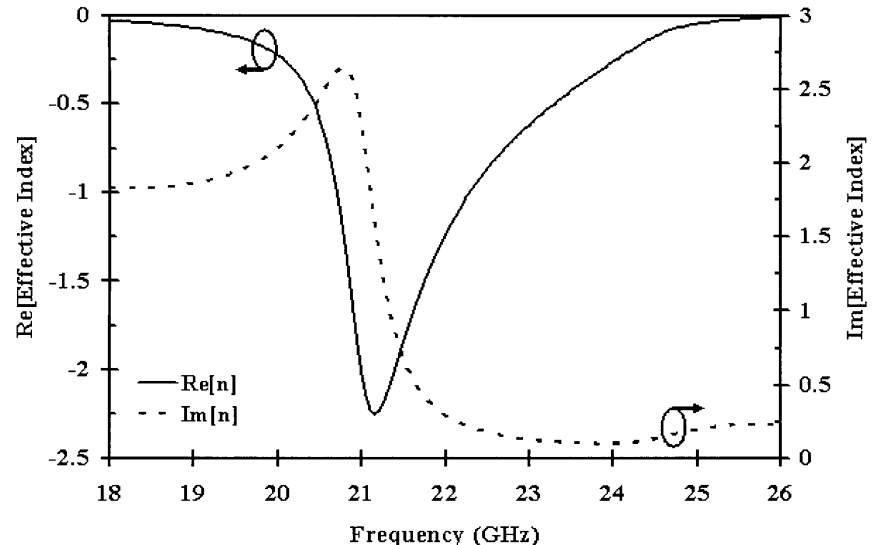

Fig. 9. Real and imaginary parts of the effective index of refraction for meta-material. The parameters used in obtaining this figure and the remaining figures in this section are: $\omega_{\mathrm{eo}}=0, \omega_{\mathrm{mo}}=2 \pi \times 21, \omega_{\mathrm{ep}}=2 \pi \times 28$, $\omega_{\mathrm{mp}}=2 \pi \times 24.5(\mathrm{GHz}), \gamma_{e}=1.6 \times 10^{9}$, and $\gamma_{m}=4 \times 10^{9}(1 / \mathrm{s})$, respectively.

radiating source, while the phase velocity is negative and points toward the source [27], [37], [39]. While a full treatment of the presence of positive and negative group velocities in meta-materials must rely on many considerations [40], [41], here, a simple model is used to clarify some aspects of the problem.

Consider a medium having an effective electric and magnetic responses characterized by [37]

$$
\varepsilon=1-\frac{\omega_{\mathrm{ep}}^{2}-\omega_{\mathrm{eO}}^{2}}{\omega^{2}-\omega_{\mathrm{eo}}^{2}-j \gamma_{e} \omega}
$$

and by

$$
\mu_{\mathrm{eff}}=1-\frac{\omega_{\mathrm{mp}}^{2}-\omega_{\mathrm{mo}}^{2}}{\omega^{2}-\omega_{\mathrm{mo}}^{2}-j \gamma_{m} \omega}
$$

where $\omega_{\mathrm{ep}}, \omega_{\mathrm{mp}}$ are the electric and magnetic plasma frequencies and $\omega_{\mathrm{eO}}, \omega_{\mathrm{mo}}$ are the electric and magnetic resonance frequencies, respectively. The $\gamma_{e}$ and $\gamma_{m}$ are the phenomenological electric and magnetic damping constants. In regions for which the real parts of the effective permeability and permittivity are both negative, the index of refraction is also negative [26]. These regions are of particular interest to us. Fig. 9 shows the real and imaginary parts of the effective index calculated from $n_{\text {eff }}=\sqrt{\varepsilon_{\text {eff }}} \sqrt{\mu_{\text {eff }}}$.

To calculate the transmission coefficient through a slab of NIR material of thickness $L$, consider the geometry shown in Fig. 10. The slab is irradiated by plane waves from a source located to its left at negative $z$ values. The transmission coefficient (magnitude and phase) can then be calculated according to

$$
T(\omega)=\frac{t_{12} t_{23} e^{i k_{2} L}}{1+r_{12} r_{23} e^{i 2 k_{2} L}}
$$

where $t_{i, j}$ and $r_{i, j}$ are the Fresnel transmission and reflection coefficients corresponding to the slab boundaries and $k_{2}$ is given by

$$
k_{2}=\frac{2 \pi}{\lambda_{o}} n_{2} \cos \theta_{2} .
$$

In the following, we assume that the LHM is surrounded by vacuum $\left(n_{1}=n_{3}=1\right)$ and is illuminated at normal incidence $\left(\theta_{1}=0\right)$.

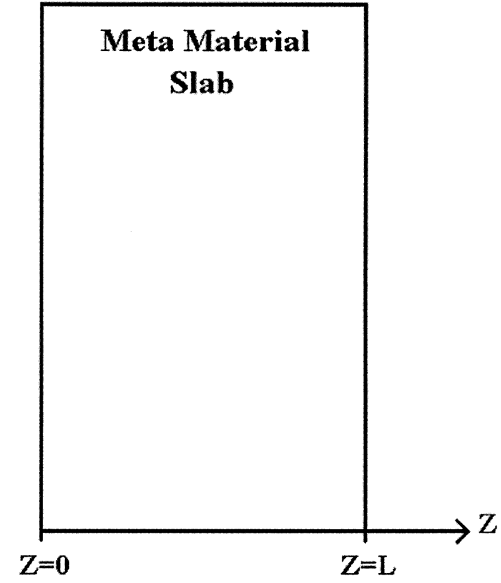

Fig. 10. A slab of meta-material illuminated by a plane wave source.

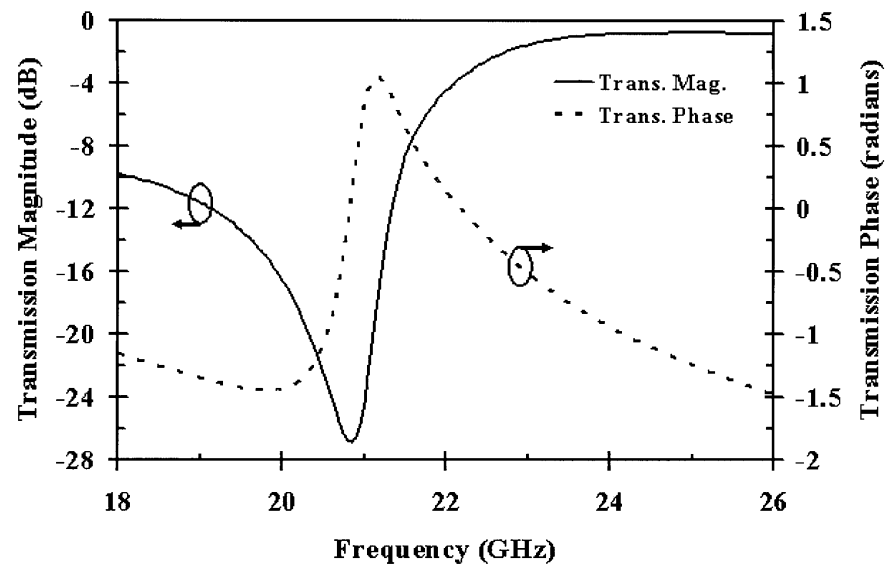

Fig. 11. Transmission magnitude and phase for a $2.5-\mathrm{mm}$ thick meta-material slab.

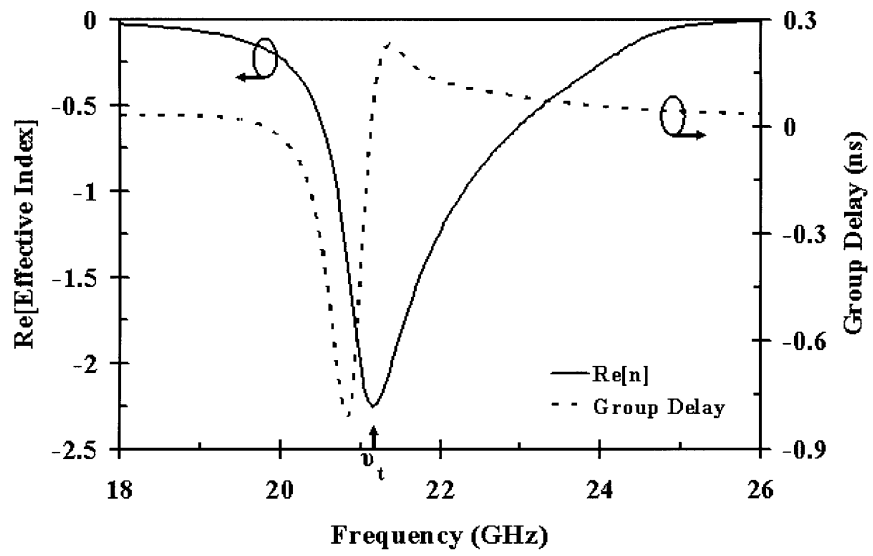

Fig. 12. Group delay and the real part of the effective index for a slab of meta-material 2.5 -mm thick

Fig. 11 shows the transmission function (magnitude and phase) for a left-handed slab 2.5 -mm thick. Note that in the vicinity of minimal transmission, corresponding to the region of anomalous dispersion, the slope of the transmission phase changes sign, consequently implying a change of the sign for the group delay $\left(\tau_{g}\right)$.

The group delay and the real part of the index are plotted in Fig. 12. From the figure it is evident that group delay, and hence the group velocity, are negative within the region of anomalous 
dispersion and are positive away from it. Note that at frequency $\nu_{t}=21.16 \mathrm{GHz}$ the group delay changes sign from negative to positive, while the refractive index remains negative. This implies that for frequencies greater than $\nu_{t}$ the group velocity is positive, whereas the phase velocity remains negative, corresponding to the backward wave propagation discussed earlier. The fact that the group velocity is positive for frequencies greater than $\nu_{t}$ can also be seen from the behavior of the index of refraction in Fig. 12. In this frequency range $\omega(d n / d \omega)$ is positive and larger than $n$, indicating a positive value for the group velocity calculated from ${ }^{5}$

$$
V_{g}=\frac{c}{n+\omega \frac{d n}{d \omega}}=\frac{c}{n_{g}}
$$

where $n_{g}$ is the group index. The existence of regions of negative group/negative phase velocities and positive group/negative phase velocities (backward waves) are also verified by the full wave simulations, the results of which will be presented elsewhere.

Since, until now [42], none of the experimental and theoretical work with the left-handed materials were carried within the region of anomalous dispersion, they all present the operation within the backward wave region. In this regard, the use of the term negative group velocity in connection with these work is incorrect. The concept of negative group velocity (negative group delay) for a medium characterized by a transmission function $T(\omega)$ has a well-defined meaning. It simply means that the peak of the output pulse is advanced in time (negative delay) such that it appears before the peak of the input pulse [18, pp. 326].

The fact that within the regions of anomalous dispersion group velocity can become negative was predicted by Garret and McCumber [43] and has been experimentally verified by some [44]-[46]. Therefore, in this respect, a reader may ask how the regions of anomalous dispersion in meta-materials may be different from the same regions in normal materials. To answer this question two observations can be made. First, consider the frequency range (or points) for which the dispersion is minimal, i.e., $d n / d \omega \approx 0$. In the vicinity of these points the group and phase velocities are approximately equal and given by

$$
V_{g} \approx V_{p}=\frac{c}{n}
$$

which is clearly negative in contrast to the case of normal materials. Second, in the region of anomalous dispersion for ordinary materials the phase velocity is positive and group velocity is negative, whereas in the region of anomalous dispersion for meta-materials both the phase and group velocities are negative. Away from the region of anomalous dispersion, the ordinary materials behave normally with both positive group and phase velocities, whereas the meta-material supports the backward waves.

Finally, note that the negative refractive index is an artificial dispersion in which the characteristics of the underlying sub-

\footnotetext{
${ }^{5}$ Equation (10) assumes perfect matching between the slab and the surrounding media, i.e., $r_{12}=r_{23}=0$. The effects of mismatches which produce positive delays will not alter the conclusions presented here. Note that the expression for group velocity in (2) automatically takes the mismatches into account.
}

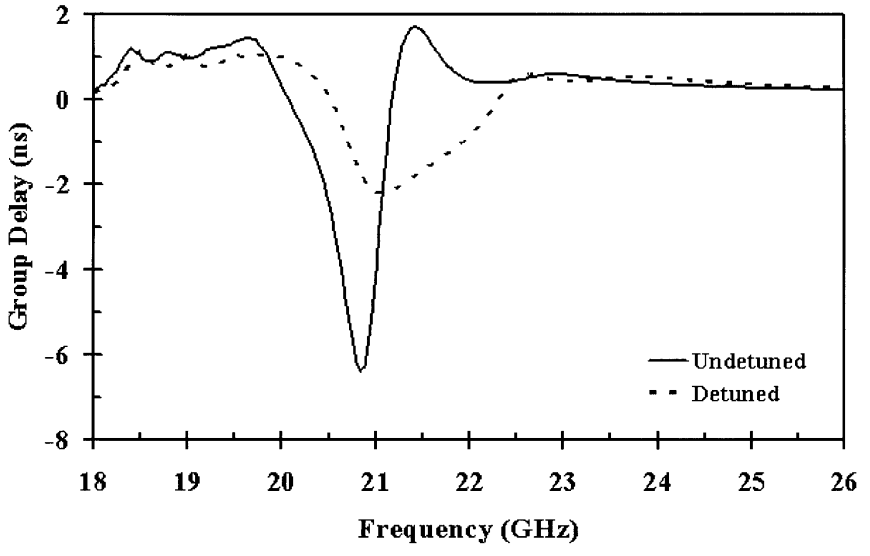

Fig. 13. The Group delay for a multilayer made of seven LHM slabs. The meta-material slab and the air spacer are each $2.5-\mathrm{mm}$ thick. In the case of detuned multilayer, the magnetic resonance of each layer has been changed by $1 \%$.

wavelength structures control the overall dispersive behavior of the media. These underlying unit cells consist of split ring resonators and conducting rods in the University of San Diego approach [26] and are loaded inductor/capacitor elements in the University of Toronto approach [2], [40], [47]. In both of these approaches, it is possible to introduce closely placed resonances (detuning) in order to increase the region of anomalous dispersion bandwidth and consequently increase the negative group delay bandwidth. As a proof of the concept, in Fig. 13 we show the case of the group delay for a 1-DPC consisted of seven alternating layers of LHM and air (solid curve). In addition, the figure also displays the group delay for the same structure, however, in which the magnetic resonance $\left(\omega_{\text {mo }}\right)$ of each slab has gradually been changed by $1 \%$ (dashed curve). As the figure indicates, for this detuned multilayer, the frequency spectrum over which the negative group delay is observed has increased by approximately $80 \%$, although the delay is less negative. In short, where in the cases of anomalous dispersion in normal materials one is more limited by the underlying atomic-molecular dipolar dispersions, for meta-materials in general, and within the region of anomalous dispersion in particular, the subwavelength macroscopic structures are analogous to the molecular dipoles, but can more easily be tailored to meet a particular design requirement.

\section{FRONT AND InFORMation In PASSIVE Media}

In Sections II-IV, we have discussed situations for which the group velocities are abnormal, i.e., are superluminal or negative. Furthermore, many theoretical and few experimental work have implied that it is possible to obtain these abnormal velocities without the attenuation observed here [11], [13], [44]-[46], [48]. Therefore, it is natural to ask whether or not these abnormal behaviors are consistent with the requirements of special relativity, which demands no information can be transmitted faster than the speed of light in vacuum. To answer this question, we must distinguish between the requirements of the first principles (Einstein Causality), and what a "practical" detection system may use in order to extract the information conveyed by an electromagnetic pulse or series of pulses. This section deals 
with the former aspect of the problem. Here, it is argued that superluminal propagation of the envelope does not imply superluminal transmission of information, since under no circumstances does the so-called "front" velocity exceed the speed of light in vacuum. This means that the presence of genuine information should not be associated with the pulse maximum or half-maximum, but must be related to the points of nonanalyticity of the information carrying signal.

When discussing the "front" we must emphasize the fact that any physically realizable signal is restrictively time limited. In other words, any electromagnetic signal generated must have a beginning in time (i.e., a "front"). One can then always point to a time prior to which the signal did not exist. For example, no physically acceptable model can imply that the wave packets displayed in Figs. 6 and 7 existed prior to the experimenter pushing the button to discharge the BWO capacitance bank. In fact, in the general discipline of signal processing, the signals discussed here appropriately carry the name of "causal signals" for which the ordinary $(-\infty,+\infty)$ limits of the convolution integral are replaced by zero and $+\infty$, as given in (11) [49, pp. $13,85]-[52$, pp. 35, 49-51]

$$
g(t)=\int_{0}^{\infty} f(\tau) h(t-\tau) d \tau .
$$

In (12), $f(\tau)$ is the input function (the incident wave packet), where $f(\tau)=0$ for $t \leq 0$ and $h(\tau)$ is the system response function. Some authors have argued physical signals posses no fronts since they are bandwidth limited [53], [54]. Clearly, accepting this definition for physically realizable signal, in which the wave packet spectrum is identically zero above and below a certain frequency (strictly bandwidth limited), would lead to the conclusion that such a signal has existed from $t=-\infty$ to $t=+\infty,{ }^{6}$ a condition unacceptable on the physical ground. An analogous situation in designing an ideal filter also exists, in which it is well known that ideal strictly bandwidth limited, low-pass, high-pass, or band-pass filters are physically unrealizable [50, pp. 125], [52, pp. 95]. Finally, any signal used for communication would have an "end-time," at which the signal level has dropped below the noise floor. However, the existence or lack thereof the "end-time" for a causal signal will not effect our conclusions regarding the luminal speed of the "front."

The proof that no signal (information) may be detected sooner than $t_{0}=x / c$ can be seen via contour integration of an expression such as (13). Equation (13) describes the field at position $x$ and time $t$ for a wave packet impinging at normal incidence on a semi-infinite medium characterized by an index of refraction, $n[18]$

$$
\begin{aligned}
& u(x, t)=\int_{-\infty}^{+\infty} \frac{2}{1+n(\omega)} A(\omega) e^{i k(\omega) x-i \omega t} d \omega \\
& A(\omega)=\frac{1}{2 \pi} \int_{-\infty}^{+\infty} u(x=0, t) e^{i \omega t} d \omega .
\end{aligned}
$$

${ }^{6}$ The reader may convince himself, by considering the Inverse Fourier Transform of a frequency-domain Rectangular function, which produces a Sinc function in the time domain.
Transforming the integral in (13) into the complex domain and closing the contour over the upper-half-plane along with the requirement that the medium characterized by $n$ is causal and that the incident wave packet has a "front" are sufficient conditions to show that the value of the integral is identically zero for $t \leq t_{0}=x / c$, or equally for velocities $V=x / t>c$. The condition that the medium characterized by $n$ is causal means that for this medium the effect cannot proceed the cause. Mathematically this is expressed as $G(\tau)=0$ for $\tau<0$, where $G(\tau)$ is the susceptibility kernel given by

$$
G(\tau)=\frac{1}{2 \pi} \int_{-\infty}^{+\infty}\left[\frac{\varepsilon(\omega)}{\varepsilon_{0}}-1\right] e^{-i \omega \tau} d \omega .
$$

The importance of the points of nonanalyticity as the "true" conveyers of information becomes clear by the following thought experiment. Suppose a noiseless superluminal medium (channel) is designed in which, while the peak of a well-behaved wave packet is shifted to earlier times, its amplitude remains unchanged. One may think of such medium as a combination of the passive and active structure described here and in [45], [46]. We then use a set of two pulses, having the maximum values of High $(\mathrm{H})$ and Low $(\mathrm{L})$, to convey a binary information. For example, it is hot $(\mathrm{H})$ or it is cold (L). If we decide to present the information carrying signal as a truly analytical function such as Gaussian, then by definition our Gaussian posses infinite number of derivatives and as such is granted a Taylor expansion about any one point in time. In other words, given a point and a small neighborhood, we can correctly predict the future behavior of the pulse including the maximum amplitude of our Gaussian ( $\mathrm{H}$ or $\mathrm{L}$ ) or extrapolate to the past and describe the wave packet behavior at an earlier time. However, as stated earlier a true Gaussian extending from $(-\infty,+\infty)$ is not a causal signal, whereas a modified Gaussian having a "front" can be used to present our causal information carrying signal. Now, let us repeat or analytical continuation argument for our causal Gaussian function. The question we like to answer is the following. What is the earliest time interval from which we can correctly predict the future value of our information carrying signal ( $\mathrm{H}$ or $\mathrm{L}$ ). For this noiseless channel, the earliest possible time is $t=0^{+}$, since $t=0$ is by definition a point of nonanalyticity for which the Taylor expansion does not exist. Therefore, in this sense, the genuine information regarding the correct value of our causal signal $(\mathrm{H}$ or $\mathrm{L})$ is contained within the time interval beginning with $t=0$ (the "front") and times immediately following it. In a practical noisy channel, the duration of this time interval depends on many experimental conditions such as the channel noise, the signal amplitude, the detection system, the behavior of the signal at the turn-on (the order of the discontinuous derivative), etc. Times immediately after $t=0$ are the beginning of the field oscillations known as the Sommerfeld and Brillouin forerunner, a subject that deserves its own exclusive consideration.

\section{CONCLUSION}

A frequency-domain setup is used to measure the group delay and hence the group velocity at frequencies within the 1-DPC stop band. It is observed that for these frequencies, the group 
delay (while positive) is less than the length of the 1-DPC divided by $c$. This implies a superluminal group velocity for frequencies tuned to the stop band of the structure. To directly verify these results, a time-domain setup is used to measure the propagation time and hence the group velocity of a single electromagnetic wave packet tunneling through a 1-DPC. Once again, it is observed that the tunneling pulse, while preserving its overall shape, travels faster than the companion wave packet traversing the same distance in air (vacuum). Furthermore, with recent interests in media having a negative index of refraction, and the confusion surrounding the existence and the meaning of negative group velocity in such materials, this subject is considered in some detail. In light of the unorthodox nature of the superluminal behavior, the relation between the observed phenomenon and the requirements of Einstein causality is investigated. It is shown that under all circumstances the "front" velocity is exactly luminal, and there are no inconsistencies with the requirements of causality.

\section{REFERENCES}

[1] M. Mojahedi, E. Schamiloglu, F. Hegeler, and K. J. Malloy, "Time-domain detection of superluminal group velocity for single microwave pulses," Phys. Rev. E (Statistical Physics, Plasmas, Fluids, and Related Interdisciplinary Topics), vol. 62, pp. 5758-5766, 2000.

[2] M. Mojahedi, E. Schamiloglu, K. Agi, and K. J. Malloy, "Frequency domain detection of superluminal group velocities in a distributed Bragg reflector," IEEE J. Quantum Electron., vol. 36, pp. 418-424, 2000.

[3] A. Ranfagni and D. Mugnai, "Anomalous pulse delay in microwave propagation : A case of superluminal behavior," Phys. Rev. E, vol. 54, pp. 5692-5696, 1996.

[4] A. Ranfagni, D. Mugnai, P. Fabeni, and G. P. Pazzi, "Delay-Time measurements in narrowed wave-guides as a test of tunneling," Appl. Phys. Lett., vol. 58, pp. 774-776, 1991.

[5] D. Mugnai, A. Ranfagni, and L. Ronchi, "The question of tunneling time duration : A new experimental test at microwave scale," Phys. Lett. A, vol. 247 , pp. 281-286, 1998.

[6] A. Enders and G. Nimtz, "Photonic-Tunneling experiments," Phys. Rev. B, vol. 47, pp. 9605-9609, 1993.

[7] _ , "On superluminal barrier traversal," J. De Physique I, vol. 2, pp. 1693-1698, 1992.

[8] C. Spielmann, R. Szipocs, A. Stingl, and F. Krausz, "Tunneling of optical pulses through photonic band-gaps," Phys. Rev. Lett., vol. 73, pp. 2308-2311, 1994.

[9] A. M. Steinberg and R. Y. Chiao, "Subfemtosecond determination of transmission delay times for a dielectric mirror (photonic band-Gap) as a function of the angle of incidence," Phys. Rev. A, vol. 51, pp. 3525-3528, 1995.

[10] A. M. Steinberg, P. G. Kwiat, and R. Y. Chiao, "Measurement of the single-photon tunneling time," Phys. Rev. Lett., vol. 71, pp. 708-711, 1993.

[11] R. Y. Chiao and A. M. Steinberg, "Tunneling times and superluminality," Progress Optics, vol. 37, pp. 345-405, 1997.

[12] E. L. Bolda, R. Y. Chiao, and J. C. Garrison, "Two theorems for the group velocity in dispersive media," Phys. Rev. A, vol. 48, pp. 3890-3894, 1993.

[13] R. Y. Chiao, "Superluminal (but causal) propagation of wave-packets in transparent media with inverted atomic populations," Phys. Rev. A, vol. 48, pp. R34-R37, 1993.

[14] E. L. Bolda, J. C. Garrison, and R. Y. Chiao, "Optical pulse-propagation at negative group velocities due to a nearby gain line," Phys. Rev. A, vol. 49, pp. 2938-2947, 1994

[15] R. Y. Chiao and J. Boyce, "Superluminality; parelectricity; and Earnshaws theorem in media with inverted populations," Phys. Rev. Lett., vol. 73, pp. 3383-3386, 1994.

[16] L. Brillouin, Wave Propagation and Group Velocity. New York: Academic, 1960.

[17] C. A. Balanis, Advanced Engineering Electromagnetics. New York: Wiley, 1989.
[18] J. D. Jackson, Classical Electrodynamics, 3rd ed. New York: Wiley, 1998

[19] G. C. Giakos and T. K. Ishii, "Energy propagation with phase velocity in a waveguide," Microwave Opt. Technol. Lett., vol. 4, pp. 128-31, 1991.

[20] - "Anomalous microwave propagation in open space," Microwave Opt. Technol. Lett., vol. 4, pp. 79-81, 1991.

[21] T. K. Ishii and G. C. Giakos, "Transmit radio messages faster than light," Microwaves and RF, vol. 30, pp. 114-119, 1991.

[22] A. Ranfagni, P. Fabeni, G. P. Pazzi, and D. Mugnai, "Anomalous pulse delay in microwave propagation : A plausible connection to the tunneling time," Phy. Rev. E, vol. 48, pp. 1453-1460, 1993.

[23] A. Enders and G. Nimtz, "Evanescent-mode propagation and quantum tunneling," Phys. Rev. E, vol. 48, pp. 632-634, 1993.

[24] V. G. Veselago, "The electrodynamics of substances with simultaneously negative values of $\varepsilon$ and $\mu$," Soviet Phys. USPEKHI, vol. 10, pp. 509-514, 1968

[25] R. A. Shelby, D. R. Smith, S. C. Nemat-Nasser, and S. Schultz, "Microwave transmission through a two-dimensional, isotropic, left-handed metamaterial," Appl. Phys. Lett., vol. 78, pp. 489-91, 2001.

[26] D. R. Smith, W. J. Padilla, D. C. Vier, S. C. Nemat-Nasser, and S. Schultz, "Composite medium with simultaneously negative permeability and permittivity," Phys. Rev. Lett., vol. 84, pp. 4184-7, 2000.

[27] D. R. Smith and N. Kroll, "Negative refractive index in left-handed materials," Phys. Rev. Lett., vol. 85, pp. 2933-6, 2000.

[28] P. M. Valanju, R. M. Walser, and A. P. Valanju, "Wave refraction in negative-index media: Always positive and very inhomogeneous," Phys. Rev. Lett., vol. 88, pp. $187401 / 1-4,2002$.

[29] S. Ramo, J. R. Whinnery, and T. Van Duzer, Fields and Waves in Communication Electronics, 3rd ed. New York, Toronto: Wiley, 1994.

[30] Applying the HP 8510 TRL Calibration for Non-Coaxial Measurements, Product Note 8510-8A, 1992.

[31] Microwave Engineering, 2nd ed., Wiley, New York, 1998.

[32] HP 8719D/8720D/8722D Network Analyzer, User's Guide 08720-90288, 1997.

[33] M. Mojahedi, "Superluminal group velocities and structural dispersion," in EECE Albuquerque: Univ. New Mexico, 1999.

[34] W. H. Press, Numerical Recipes in FORTRAN : The Art of Scientific Computing, 2nd ed., Cambridge, U.K., 1992.

[35] L. D. Landau, E. M. Lifshittz, and L. P. Pitaevski, Electrodynamics of Continuous Media, 2nd, rev. and enl. ed. Oxford, Oxfordshire, 1984.

[36] L. Brillouin, Wave Propagation in Periodic Structures; Electric Filters and Crystal Lattices, 1st ed. New York: McGraw-Hill, 1946.

[37] R. A. Shelby, D. R. Smith, and S. Schultz, "Experimental verification of a negative index of refraction," Science, vol. 292, pp. 77-9, 2001.

[38] J. B. Pendry, "Negative refraction makes a perfect lens," Phys. Rev. Lett., vol. 85, pp. 3966-9, 2000.

[39] V. G. Veselago, "Properties of materials having simultaneously negative values of the dielectric $(\epsilon)$ and the magnetic $(\mu)$ susceptibilities," Soviet Phys.-Solid State, vol. 8, pp. 2854-2856, 1967.

[40] M. Mojahedi, S. O., W. J., and E.G. V., "Pulse propagation and negative group delay in metamaterials," in Progress in Electromagnetics Res. Symp., Cambridge, MA, 2002

[41] - "Superluminal velocities in causal media," in KITP Miniprogram on Quantum Optics, Santa Barbara, CA, 2002.

[42] O. Siddiqui, M. Mojahedi, and G. V. Eleftheriades, "Periodically loaded transmission line with effective negative refractive index and negative group velocity," IEEE Trans. Antennas Propagation, Special Issue Metamaterials, 2002, to be published.

[43] C. G. B. Garrett and D. E. McCumber, "Propagation of a gaussian light pulse through an anomalous dispersion medium," Phys. Rev. A, vol. 1, pp. 305-313, 1970.

[44] S. Chu and S. Wong, "Linear pulse-propagation in an absorbing medium," Phys. Rev. Lett., vol. 48, pp. 738-741, 1982.

[45] L. J. Wang, A. Kuzmich, and A. Dogariu, "Gain-assisted superluminal light propagation," Nature, vol. 406, pp. 277-9, 2000.

[46] M. W. Mitchell and R. Y. Chiao, "Negative group delay and fronts in a causal system : An experiment with very-low frequency bandpass-amplifiers," Phys. Lett. A, vol. 230, pp. 133-138, 1997.

[47] G. V. Eleftheriades, A. K. Iyer, and P. C. Kremer, "Planar negative refractive index media using periodically $L-C$ loaded transmission lines," IEEE Trans. Microwave Theory and Techniques, vol. 50, pp. 2702-2712, Dec. 2002.

[48] R. Y. Chiao, A. E. Kozhekin, and G. Kurizki, "Tachyonlike excitations in inverted 2-Level media," Phys. Rev. Lett., vol. 77, pp. 1254-1257, 1996.

[49] A. Papoulis, The Fourier Integral and its Applications. New York: McGraw-Hill, 1962. 
[50] C. D. McGillem and G. R. Cooper, Continuous and Discrete Signal and System Analysis, 2nd ed. New York: Holt Rinehart and Winston, 1984.

[51] F. G. Stremler, Introduction to Communication Systems, 2nd ed. Reading, MA: Addison-Wesley, 1982.

[52] A. B. Carlson, Communication Systems : An Introduction to Signals and Noise in Electrical Communication, 3rd ed. New York: McGraw-Hill, 1986.

[53] W. Heitmann and G. Nimtz, "On causality proofs of superluminal barrier traversal of frequency band-limited wave-packets," Phys. Lett. A, vol. 196, pp. 154-158, 1994.

[54] G. Nimtz, "Evanescent modes are not necessarily einstein causal," Eur. Phys. J. B, vol. 7, pp. 523-525, 1999.

M. Mojahedi (S'97-M'98) received the Ph.D. degree from the Center for High Technology Materials (CHTM), University of New Mexico (UNM), in December 1999.

He worked as a Research Assistant Professor at the CHTM. In August 2001, he joined the faculty of the Electrical and Computer Engineering at the University of Toronto, Ontario, Canada. His research interests include matter wave interactions, abnormal velocities, meta-materials, photonic crystals, dispersion engineering, quantum dots and wells lasers, fundamental electromagnetic theory, periodic structures, macro and nano-scale microwaves, and photonic systems.

Dr. Mojahedi was the recipient of the Popejoy Award for outstanding doctoral dissertation in Physics and Engineering, UNM, for the years 1997 to 2000.

K. J. Malloy (S'76-S'78-M'79-S'80-M'82-S'83-M'83) photograph and biography not available at the time of publication.
G. V. Eleftheriades ( $\left.\mathrm{S}^{\prime} 86-\mathrm{M}^{\prime} 88-\mathrm{S}^{\prime} 88-\mathrm{M}^{\prime} 95-\mathrm{SM}^{\prime} 02\right)$ received the $\mathrm{Ph} . \mathrm{D}$. and M.S.E.E. degrees in electrical engineering from the University of Michigan, Ann Arbor, in 1993 and 1989, respectively. He received a diploma (with distinction) in electrical engineering from the National Technical University of Athens, Greece, in 1988 .

From 1994 to 1997, he was with the Swiss Federal Institute of Technology in Lausanne, where he was engaged in the design of millimetre and sub-millimetre-wave receivers and in the creation of fast CAD tools for planar packaged microwave circuits. In 1997, he joined the Department of Electrical and Computer Engineering at the University of Toronto where he is now an Associate Professor. He has authored or coauthored more than 80 articles in refereed journals and conference proceedings. Currently, he is leading a Group of about 14 graduate students in the areas of negative refractive index metamaterials, IC antennas and components for broad-band wireless communications, novel antenna beam-steering techniques, low-loss silicon micromachined components, submillimeter-wave radiometric receivers, and electro-magnetic design for high-speed digital circuits.

Dr. Eleftheriades was a corecipient of the Best Paper Award at the 6th International Symposium on Antennas (JINA), France, 1990. More recently, his graduate students won Best Student Paper Awards in the 2000 Antenna Technology and Applied Electromagnetics Symposium, the 2002 IEEE International Microwave Symposium, and the 2002 International Symposium on Antennas and Propagation. He received the Ontario Premier's Research Excellence Award, in 2001.

J. Woodley (M'02) photograph and biography not available at the time of publication.

R. Y. Chiao, photograph and biography not available at the time of publication. 